\title{
Budding Yeast Forms on Peripheral Blood Smear: An Intriguing Finding
}

\author{
Poojan Agarwal $^{1} \cdot$ Narender Tejwani $^{1} \cdot$ Neelam Sachdeva $^{1} \cdot$ Anurag Mehta $^{1}$
}

Received: 6 June 2017 / Accepted: 14 July 2017 / Published online: 26 July 2017

(C) Indian Society of Haematology \& Transfusion Medicine 2017

54 year old female, case of metastatic carcinoma breast presented to the emergency in semi-comatose state. Investigations revealed normal renal and mildly deranged liver functions. Hemogram done from central line sample showed $\mathrm{Hb} 9.7$ of $\mathrm{g} \%$, neutrophilic leukocytosis and adequate platelets. Peripheral blood smear (PBS) showed numerous intracellular (within neutrophils and monocytes) yeast forms (Fig. 1) which were morphologically difficult to differentiate from histoplasma species. The organisms were positive for PAS and Gomori methenamine silver (GMS) stain and negative for mucicarmine \& India ink preparation. Fresh blood and tracheal secretion culture revealed yeast within $24 \mathrm{~h}$ of incubation. Subculture streaking revealed dry, dull creamy white heaped up colonies of Candida. Identification was done on Vitec-2 which revealed Candida tropicalis. The patient succumbed to her metastatic disease and bacterial sepsis developing during ICU stay 7 days later.

Candida is commonest cause of blood stream fungal infection but still it is rarely reported in PBS [1]. This is because $1-5 \times 10^{5} \mathrm{CFU} / \mathrm{mL}$ organisms are required to detect them on PBS [2]. An awareness of morphology of these organisms is essential for identification and

Narender Tejwani

mbbsnt@gmail.com

Poojan Agarwal

poojanagarwal@gmail.com

Neelam Sachdeva

Sachdeva.neelam@rgcirc.org

Anurag Mehta

mehta.anurag@rgcirc.org

1 Department of Laboratory Medicine, Rajiv Gandhi Cancer Institute and Research Center, Rohini, Delhi, India

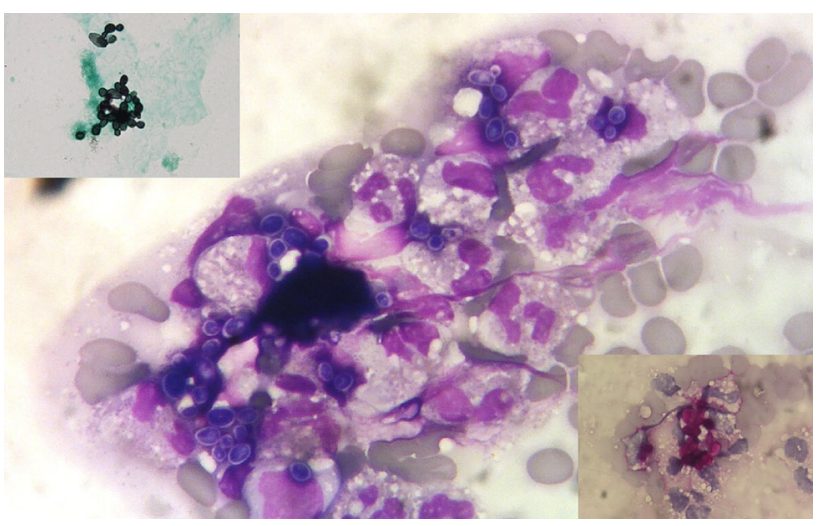

Fig. 1 Multiple intracellular yeast form of candida sp. in peripheral smear $(\times 400$, Giemsa stain $)$ positive for GMS $(\times 400$, left upper inset $)$ and periodic acid Schiff $(\times 400$, right lower inset $)$

differentiation from other causes of blood stream fungal infections like Histoplasma and Cryptococcus.

\section{Compliance with Ethical Standards}

Conflict of interest The authors declares that they have no conflict of interest.

Disclaimers The identity of the patient is not disclosed here in this case.

Funding The study is the retrospective analysis of the data of patients at the Rajiv Gandhi Cancer Hospital and Research Institute. No funding received.

\section{References}

1. Hirai Y, Asahata S, Ainoda Y, Fujita T, Miura H, Hizuka N et al (2015) Candidemia diagnosed from peripheral blood smear: case report and review of literature 1954-2013. Mycopathologia 180(1-2):111-116

2. Branda JA, Ferraro MJ, Kratz A (2007) Sensitivity of peripheral blood smear review for the diagnosis of Candida fungemia. Arch Pathol Lab Med 131(1):97-101 